\title{
ANÁLISIS DE LA COMPLETITUD DE PRAXEOLOGÍAS PROPUESTAS A ESTUDIAR EN TORNO A LA LÓGICA EN LA FORMACIÓN DE PROFESORES DE MATEMÁTICA
}

\section{ANALYSIS OF THE COMPLETITUDE OF PRAXEOLOGIES PROPOSED TO STUDY AROUND LOGIC IN THE TRAINING OF MATHEMATICS TEACHERS}

\section{Oscar Abel Cardona Hurtado ${ }^{1}$}

Universidad del Tolima e Institución Educativa Liceo Nacional de Ibagué

\section{Ana Rosa Corica ${ }^{2}$}

Consejo Nacional de Investigaciones

Científicas y Técnicas

$1 \quad$ Profesional en Matemáticas con Énfasis en Estadística y Especialista en Matemáticas Avanzadas de la Universidad del Tolima, Colombia; Magister en Matemáticas Aplicadas de la Universidad EAFIT, Colombia; Estudiante de Doctorado en Enseñanza de las Ciencias, de la UNCPBA, Argentina. Catedrático de la Universidad del Tolima y Docente de Matemáticas de la Institución Educativa Liceo Nacional, de Ibagué. Investigador en Lógica Matemática y en Educación Matemática. Correo: oach76@hotmail.com. Orcid: https://orcid.org/0000-0003-0907-5706

2 Doctora en Ciencias de la Educación por la UNC en Argentina. Licenciada en Educación Matemática y Profesora en Matemática y Física por la UNCPBA. Investigadora Adjunta del CONICET. Investigadora del NIECyT. Profesora Adjunta de la Facultad de Ciencias Exactas de la UNCPBA, Tandil, Buenos Aires, Argentina. Correo: acorica@exa.unicen.edu.ar. Orcid: https://orcid.org/0000-0002-3583-6081

\section{RESUMEN}

En este trabajo se presentan resultados de una investigación que se ubica en la problemática de la formación de profesores en lógica matemática. Como referencial teórico se adopta a la Teoría Antropológica de lo Didáctico. La investigación se desarrolló en dos grupos de estudiantes de profesorado en matemática de una universidad colombiana. Se analizaron las prácticas de los docentes que orientaron temas 
relativos a cálculo proposicional y cálculo de predicados, y se examinó el material propuesto para el estudio. En particular, en este trabajo se reportan resultados del análisis del material propuesto por los docentes; se analizaron los componentes praxeológicos que se identificaron en las tareas resueltas y las tareas formuladas para ser resultas por los estudiantes. Los principales resultados de la investigación indican que tanto las tareas resueltas en el material como las tareas propuestas para su resolución, integran praxeologías con bajo grado de completitud.

\section{PALABRAS CLAVE:}

Formación, Profesor, Lógica, Teoría

Antropológica de lo Didáctico

\section{ABSTRACT}

In this work are presented results of an investigation that is located in the problematic of the formation of teachers in mathematical logic. As a theoretical reference, the Anthropological Theory of Didactics is adopted. The research was developed in two groups of mathematics teacher students from a Colombian university. The practices of the teachers who guided topics related to propositional calculus and predicate calculus were analyzed, and the material proposed for the study was examined. In particular, this work reports the results of the analysis of the material proposed by the teachers; the praxeological components that were identified in the solved tasks and the tasks formulated to be solved by the students were analyzed. The main results of the research indicate that both the tasks solved in the material and the tasks proposed for their resolution, integrate praxeologies with a low degree of completeness.

KEY WORDS: Training, Teacher, Logic, Anthropological Theory of Didactics

\section{INTRODUCCIÓN}

La lógica se relaciona con el estudio de los métodos y los principios indispensables con el fin de distinguir el razonamiento correcto del incorrecto, para lo cual se han desarrollado métodos y técnicas (Del Callejo, Del Callejo y Canal, 2016). Copi y Cohen (2013) indican que los seres humanos utilizamos la lógica para obtener razones justificables ya sea en la vida privada, en la ciencia o en la política. La sociedad actual proporciona a los individuos grandes cantidades de información principalmente por medios como los dispositivos electrónicos, y esto demanda de respuestas oportunas y razonables. La lógica matemática como ciencia útil para establecer la validez de argumentos, proporciona recursos para la toma de decisiones con fundamento en la razón y no en emociones o creencias (Cardona y Corica, 2020; Vázquez, 2020).

El cálculo proposicional (en adelante CP) y el cálculo de predicados (en lo sucesivo CDP) son ámbitos de la lógica matemática que permiten estudiar relaciones entre las proposiciones, los conectivos lógicos y los cuantificadores. La representación simbólica de enunciados y de argumentos de la vida cotidiana, permite aprovechar el poder de la lógica matemática en la solución de problemas que aquejan a diario a las personas. Asimismo, el CP y el CDP juegan un papel central en la demostración matemática, posibilitando entre otras cosas, probar la equivalencia entre el método de demostración directa y métodos de demostración indirecta. También, estos ámbitos se caracterizan por sus aplicaciones en áreas de la informática como la inteligencia artificial, la programación y el diseño de circuitos lógicos. Particularmente, la inteligencia artificial tiene que ver con que las computadoras puedan mecanizar tareas que el hombre hace de manera rutinaria; estas tareas requieren de una formalización que se realiza empleando el CP y el CDP (Fernández y Sáez, 1995). 
Teniendo en cuenta la utilidad del CP y el CDP en variados contextos de la vida cotidiana de las personas, se considera fundamental que estos ámbitos sean enseñados en las instituciones educativas en los distintos niveles. También, aspectos relacionados con la enseñanza de estos campos de la lógica matemática deberían ser objeto de investigación por parte de la comunidad académica especializada. No obstante, no se encontraron investigaciones enfocadas en analizar la manera como se enseña CP y CDP a estudiantes de profesorado en matemática. Considerando que los docentes son actores centrales en los procesos académicos, resulta pertinente conocer en particular, cómo están siendo formados los futuros profesores en temas relativos a CP y CDP.

Este trabajo refiere a una investigación que se ubica en la problemática relacionada con la formación de profesores de matemática. Se analizaron las prácticas de profesores universitarios que impartieron clases a estudiantes de profesorado en matemática, en las que se estudiaron temas relativos a lógica matemática Se adoptó como marco teórico a la Teoría Antropológica de lo Didáctico propuesta por Chevallard (1999). En este referencial teórico se considera fundamental el estudio del saber propuesto para ser enseñado. Dicho saber se materializa en nociones que son seleccionadas del saber producido por especialistas en una disciplina y transformado para convertirlo en saber escolar, y propiciar la apropiación de este por parte de los estudiantes. En este trabajo se reportan resultados del análisis de los saberes propuestos para ser enseñados a profesores de matemática en formación en una universidad colombiana. En particular, los indicadores de completitud propuestos por Fonseca (2004) y Lucas (2010) permitieron examinar el grado de completitud de las praxeologías propuestas para el estudio de temas relacionados con CP y CDP en el marco de un curso impartido.

\section{MARCO TEÓRICO}

La Teoría de Transposición Didáctica (Chevallard, 1991) supone que para que un saber particular producido por especialistas en un ámbito (saber sabio), sea enseñado en una institución educativa, es necesario someterlo a modificaciones adaptativas que posibiliten su reconstrucción en el seno de la comunidad educativa (Bosch y Gascón, 2007). Dicha teoría distingue distintas clases de saberes, que surgen a partir de las transformaciones realizadas al saber sabio. El desarrollo de esta teoría ha puesto en evidencia que las diferentes formas de manipulación social de la matemática no pueden ser estudiadas de manera independiente (Gascón, 1998). El saber sabio es aquel propio de una minoría que cuenta con la formación necesaria para comprenderlo. Este saber es producto de investigaciones científicas y se encuentra registrado en textos académicos no comprensibles para la mayoría de miembros de la comunidad educativa. El saber a enseñar procede de una selección del saber sabio y una adaptación, que generalmente se registra en libros de texto, en diseños curriculares o en otros documentos propios de las instituciones educativas. Chevallard (1991) establece que la noosfera es la responsable de la selección de los saberes, y también la encargada de realizar las adaptaciones requeridas con el objetivo de construir un producto adecuado para la enseñanza a los estudiantes. El saber enseñado refiere a aquel que inicialmente fue designado como saber a enseñar, y que posteriormente fue objeto de una serie de transformaciones con el fin de adaptarlo para llevarlo a las aulas, y presentarlo a los estudiantes para su apropiación. El saber aprendido alude al modelo construido por el estudiante al final del proceso de estudio. Este saber es fruto de la apropiación del saber enseñado por parte del alumno.

La Teoría de Transposición Didáctica se constituyó en el germen que le permitió años 
más tarde a Yves Chevallard proponer la Teoría Antropológica de lo Didáctico (en lo sucesivo TAD). La TAD ubica a la actividad matemática dentro de las actividades humanas y las instituciones sociales (Bosch y Gascón, 2007). La noción de praxeología (u Organización Matemática), es el constructo teórico fundamental de la TAD, surge como respuesta a un conjunto de cuestiones, y consta de dos componentes: la praxis o del saber hacer y el logos o del saber. El nivel de la praxis o del saber hacer, está compuesto por conjunto de tareas que se materializan en diferentes tipos de problemas, y de un conjunto de técnicas que se utilizan para llevar a cabo las tareas planteadas; y el nivel del logos o del saber en el que se sitúan, en un primer nivel, el discurso que describe, explica y justifica la técnica, denominada tecnología, y en un segundo nivel, la fundamentación de la tecnología, denominada teoría que asume respecto a la tecnología el mismo papel descriptivo y justificativo que el de la tecnología respecto de la técnica. Por otra parte, un género de tareas, no existe más que bajo la forma de diferentes tipos de tareas, las que se caracterizan por contar con un contenido estrechamente especificado. El concepto tipo de tareas, alude a un objeto relativamente preciso. A manera de ejemplo, al estudiar cálculo proposicional surge el género de tareas Demostrar, y a este se asocian tipos de tareas como Demostrar la conclusión producto de un conjunto de premisas o Demostrar propiedades algebraicas que satisfacen los conectivos.

La TAD distingue entre dos tipos de praxeologías: la Organización Matemática (OM) y la Organización Didáctica (OD). La primera hace alusión a la realidad matemática a estudiar, la segunda tiene que ver con la manera en que esto ocurre. Los dos aspectos son inseparables: toda OM es generada por un estudio y al mismo tiempo, todo proceso de estudio se realiza con base en una OM en construcción. Asimismo, Chevallard (1999) formuló cuatro tipos de praxeologías teniendo en cuenta el grado de complejidad de sus componentes, resultando útil para analizar los procesos didácticos institucionales. A continuación se sintetizan los tipos de praxeologías. Las Organizaciones Puntuales (OMP) se generan en la institución por lo que se considera como un único tipo de tarea y se define a partir del bloque prácticotécnico. Las Organizaciones Locales (OML) son el resultado de integrar diversas praxeologías puntuales. Las Organizaciones Regionales se obtienen mediante la coordinación, articulación y posterior integración de diversas praxeologías Locales a una teoría matemática en común. Finalmente, las Organizaciones Globales surgen al agregar varias praxeologías regionales a partir de la integración de diferentes teorías. Para que una OML goce de alto grado de completitud, se propone organizar el estudio con base en momentos didácticos Chevallard (1999), y las características del producto resultante se analizan mediante indicadores. Los rasgos del producto resultante se examinan empleando ocho indicadores matemáticos de completitud formulados por Fonseca (2004) y Lucas (2010).

A continuación se describen los indicadores matemáticos antes mencionados y que resultan útil para el estudio que se presenta en este trabajo. OML1. Integración de tipos de tareas y existencia de tareas relativas al cuestionamiento tecnológico; dado que en una OM conviven varios tipos de tareas, dicha OM es más completa en la medida que existan menos tareas aisladas, esto es, tareas resultas mediante técnicas que no están relacionados entre sí por ningún elemento tecnológicoteórico. OML2. Diferentes técnicas para cada tipo de tareas y criterios para elegir entre ellas; una $\mathrm{OM}$ es más completa si, dada una tarea particular, se cuenta con dos o más técnicas que permiten resolverla, y criterios para elegir la técnica más adecuada. OML3. Independencia de los objetos ostensivos que sirven para representar las técnicas; una OM goza de 
mayor grado de completitud, si las técnicas son más flexibles, es decir, si dada una técnica, se cuenta con diversidad de representaciones para su aplicación. OML4. Existencia de tareas y de técnicas inversas; una OM presenta mayor grado de completitud en la medida en que cuente con algunas técnicas inversas, es decir, técnicas que hagan posible realizar las tareas inversas; por ejemplo aquellas definidas intercambiando los datos y las incógnitas de la tarea inicial. OML5. Interpretación del funcionamiento y del resultado de aplicar las técnicas; una OM es más completa si se cumple que para cada técnica asociada a la OM, existe en la misma $\mathrm{OM}$ el tipo de tareas consistente en interpretar el funcionamiento y el resultado de aplicar la técnica; lo cual implica la existencia de elementos tecnológico-teóricos útiles para realizar la interpretación. OML6. Existencia de tareas matemáticas abiertas; una OM será más completa en la medida que existan tipos de tareas matemáticas abiertas, esto es, tipos de tareas matemáticas en los que los datos y las incógnitas no están prefijados completamente de antemano. OML7. Integración de los elementos tecnológicos e incidencia sobre la práctica; el grado de completitud de OM dependerá también del grado de integración interna de los elementos que componen el entorno tecnológico-teórico y de la incidencia efectiva de estos elementos sobre la práctica. OML8. La posibilidad de perturbar la situación inicial o modificar la hipótesis del sistema para estudiar casos diferentes, permite ampliar y completar el proceso de estudio; este indicador trata sobre la posibilidad de perturbar la situación, lo cual permitiría la aparición de nuevas tareas, de nuevas técnicas, y de nuevos elementos que se sumarían al entorno tecnológico-teórico, enriqueciendo así el proceso de estudio. Se destaca que, según Fonseca (2004), no existen organizaciones matemáticas completas ni organizaciones matemáticas incompletas, se trata de una cuestión de grado de completitud, que depende del cumplimiento de los ocho indicadores; los siete primeros propuestos por Fonseca (2004) y el octavo por Lucas (2010).

\section{METODOLOGÍA}

El trabajo que se presenta es cualitativo, de corte exploratorio, descriptivo e interpretativo (Hernández, Fernández y Baptista, 2014). Se examinaron las prácticas docentes dirigidas a estudiantes de profesorado en matemática en una universidad colombiana; en las que se trataron temas alusivos a CP y a CDP. La investigación se realizó en dos grupos de estudiantes de un mismo programa universitario que realizaron el mismo curso. Cada grupo estuvo a cargo de un docente distinto. Uno de los grupos estaba conformado por 20 estudiantes y el otro por 22; la edad de los alumnos oscilaba entre 17 y 18 años. El curso se desarrolló durante 16 semanas; en cada una de ellas se realizaron dos sesiones de clase de 120 minutos. El estudio de saberes relacionados con $\mathrm{CP}$ y $\mathrm{CDP}$, correspondiente a la primera de las seis unidades descritas en el diseño curricular del curso, se llevó a cabo durante las tres primeras semanas.

En correspondencia con el marco teórico adoptado, el estudio se realizó en cuatro fases. La primera aludió a la reconstrucción de un Modelo Praxeológico de Referencia (en adelante MPR) relativo a CP y nociones básicas de CDP, que tiene que ver con una organización de los saberes a enseñar. Dicho MPR fue reconstruido por los investigadores a partir de sus conocimientos, los datos recolectados durante la investigación, consultas realizadas a especialistas y revisión bibliográfica especializada. Este constructo se caracteriza por su utilidad para el análisis de las organizaciones matemáticas encontradas a lo largo de la investigación y también para proponer praxeologías. García, Barquero, Florensa y Bosch (2019) destacan que la complejidad en la reconstrucción de un MPR demanda del estudio y del cuestionamiento de diferentes fuentes de información, como instituciones productoras del saber e 
instituciones encargadas de la enseñanza. Por otra parte, de acuerdo a Barquero, Bosch y Gascón (2013), un MPR por sus características particulares se constituye en un instrumento de emancipación didáctica, puesto que permite cuestionar la manera como las instituciones en las cuales emergen problemáticas matemáticas y/o didácticas interpretan el saber matemático. Asimismo, el MPR corresponde a un modelo alternativo que posibilita analizar y poner en entredicho paradigmas relacionados con maneras de entender un saber matemático particular (Gascón, 2014).

En la segunda etapa de la investigación se reconstruyó la Organización Matemática Propuesta a Enseñar (OMPE), relacionada con los saberes puestos en consideración para ser enseñados. Esta se confeccionó con base al material sugerido para el estudio por los profesores que orientan el curso para el estudio de temas relativos a CP y a CDP. Para la reconstrucción de la OMPE también se revisó el diseño curricular del curso, pero este resulta ser muy sintético, lo que no posibilita realizar el análisis requerido. En la tercera etapa de la investigación se reconstruyó la Organización Matemática Efectivamente Enseñada (en adelante (OMEE); esta corresponde a un estudio de lo realmente enseñado por los docentes que impartieron el curso. Cabe destacar que no siempre lo que se propone para ser enseñado en un proceso de estudio, es lo que realmente se termina enseñando. Para la reconstrucción de dicha OMEE fue necesario realizar observaciones no participantes en los dos grupos, lo que permitió recolectar la información requerida para el análisis respectivo. En la cuarta y última fase del estudio, a partir de las reconstrucciones producto de las tres primeras fases, se propuso tareas extramatemáticas provenientes de situaciones de la vida cotidiana, con el propósito de contribuir al estudio funcional de temas relativos a CP y a CDP. En el presente trabajo se hace hincapié en la descripción de la segunda fase, relacionada con la reconstrucción de la OMPE; para el análisis de esta fue fundamental el MPR reconstruido en la fase 1.

En la reconstrucción de la OMPE se examinó el libro de texto titulado Introducción a la lógica matemática cuyos autores son Patrick Suppes y Shirley Hill (Suppes y Hill, 1975); documento utilizado como único media por dos docentes que orientaron el curso, en el que enseñaron conceptos relativos a CP y a CDP, a estudiantes de profesorado en matemática en la universidad colombiana que se involucró en la investigación.

El libro analizado se compone de ocho capítulos, titulados de la siguiente manera: Simbolización de proposiciones, Inferencia lógica, Certeza y validez, Tablas de certeza, Términos predicados y cuantificadores, Especificación universal y leyes de identidad, Un sistema axiomático simple: axiomas de la adición y Generalización universal. Los dos docentes que impartieron las clases abordaron temáticas relacionadas con los capítulos 1,2 , 3,4 y 5 del libro de texto; el contenido incluido en estos cinco capítulos se considera adecuado de acuerdo al curso, teniendo en cuenta que abarca las temáticas sugeridas por la institución en el diseño curricular.

En concordancia con el referencial teórico adoptado, para el análisis del libro de texto propuesto por los docentes, se estudiaron pormenores de los capítulos; en cada uno se describió el entorno tecnológico-teórico expuesto, se analizaron los ejemplares de tarea resueltos y se examinaron las tareas propuestas para ser resueltas. Con la finalidad de estudiar en detalle las tareas propuestas para su resolución en los capítulos analizados del libro de texto, se organizaron en una tabla que se compone de las categorías que se muestran en la Tabla 1. 
Tabla 1. Categorías para analizar tareas propuestas

\begin{tabular}{|l|c|c|c|c|c|}
\hline Genero de tareas & $\begin{array}{c}\text { Tipo de } \\
\text { tareas }\end{array}$ & Ejemplar resuelto & Técnica & $\begin{array}{c}\text { Bloque } \\
\text { tecnológico- } \\
\text { teórico }\end{array}$ & I MC \\
\hline
\end{tabular}

Fuente: Elaboración propia

La tabla que se indica en la Tabla 1 consta de seis columnas. Cada columna refiere a las categorías formuladas. En la primera columna se indican los géneros de tareas a los que refieren los tipos de tareas identificados en el texto. Los tipos de tareas son exhibidos en la segunda columna. En la tercera columna se indican ejemplares de tarea correspondientes a los tipos de tareas identificados y las técnicas explicitas en el libro de texto para ser resueltos. En la cuarta columna se describen las técnicas que fueron empleadas para solucionar las tareas de la columna anterior. En la quinta columna se identifica el bloque tecnológico - teórico inmediato que justifica la manera de hacer propuesta en el texto para el tipo de tarea identificado. Finalmente, en la sexta columna se señalan los indicadores matemáticos de completitud de una OML (IMC) identificados en el estudio de la tarea respectiva. En el presente estudio, con fundamento en los ocho indicadores antes mencionados se examina el grado de completitud de las praxeologías propuestas en el libro de texto propuesto por los dos docentes que orientaron temas alusivos a CP y CDP a estudiantes para profesor de matemática.

\section{EL MODELO PRAXEOLÓGICO DE REFERENCIA}

El MPR alusivo al estudio de CP y nociones básicas de CDP fue diseñado por los investigadores utilizando sus conocimientos, y recurriendo a recursos bibliográficos y consultas a expertos. El estudio del fenómeno de transposición didáctica puso de presente que es tarea fundamental de los investigadores en didáctica buscar que sus estudios se liberen de los códigos establecidos por las instituciones; el didacta requiere estudiar de manera crítica los modelos epistemológicos dominantes en las instituciones (Chevallard, 1997; Gascón, 2014). Cabe destacar que los alcances de un MPR van más allá de cuestionar modelos dominantes, este instrumento también favorece la construcción de modelos alternativos (García y Sierra, 2015). Así, el MPR propuesto posibilita analizar y cuestionar la OMPE reconstruida a partir del material propuesto para el estudio por ambos profesores.

La descripción de un MPR suele hacerse mediante una red de preguntas y respuestas que tienen estructura praxeológica, constituyendo una importante herramienta didáctica. Su elaboración, en torno a un ámbito particular, conduce a la formulación de preguntas didácticas que cobran importancia si se pretende avanzar en la modificación de los métodos de enseñanza tradicional, en los cuales, las preguntas no juegan un papel central. Se destaca que el MPR debe ser considerado como una hipótesis provisional, lo cual implica que es susceptible de ser revisado y modificado constantemente (Otero, Fanaro, Corica, Llanos, Sureda y Parra, 2013).

El MPR que se describe de manera sintética en el presente apartado, gira en torno a CP y nociones básicas de CDP. EI CP es aquella rama de la lógica matemática que trata de las relaciones entre proposiciones y conectivos lógicos. El CDP es una generalización del CP que cubre limitaciones expresivas de este último (Garrido, 2005). El CP es insuficiente para estudiar la estructura interna de las 
proposiciones. De tal suerte que es necesario recurrir al CDP que incluye entre sus componentes más destacados al cuantificador universal y al cuantificador existencial, los cuales permiten estudiar la composición de los enunciados que suelen ser estudiados en el CP, posibilitando conocer acerca de los elementos de un conjunto que satisfacen cierta propiedad y las relaciones entre los mismos.

El CP y el CDP como dominios de la lógica matemática, se consideran en el MPR desde dos facetas: por un lado la representación simbólica de enunciados y argumentos entendidos como expresiones de la vida cotidiana, y la posibilidad de establecer la verdad o falsedad de estos; por otro lado, el estudio de proposiciones, conectivos y cuantificadores, como objetos matemáticos que gozan de propiedades y satisfacen relaciones propias de este ámbito de la ciencia.

EI MPR se origina a partir de la cuestión generatriz $Q_{0}$ : ¿Cómo establecer la validez de un razonamiento? De $Q_{0}$ se derivan las preguntas $Q_{1}$ : ¿Cómo establecer el valor de verdad de una proposición relativa al CP? Que adule a saberes relativos a $C P$, y $Q_{2}$ : ¿Cómo establecer el valor de verdad de una proposición relativa al CDP? Que hace referencia a temáticas relativas a CDP. Se considera que $Q_{0}$ corresponde a un interrogante planteado en sentido fuerte; en el MPR que se describe se aborda desde los dos ámbitos de la lógica matemática mencionados. En la Imagen 1 se muestran las tres cuestiones generatrices que dan origen al MPR, las OM asociadas a estas y las tareas relacionadas con estas últimas; también se indican relaciones entre las preguntas, las OM y las tareas.

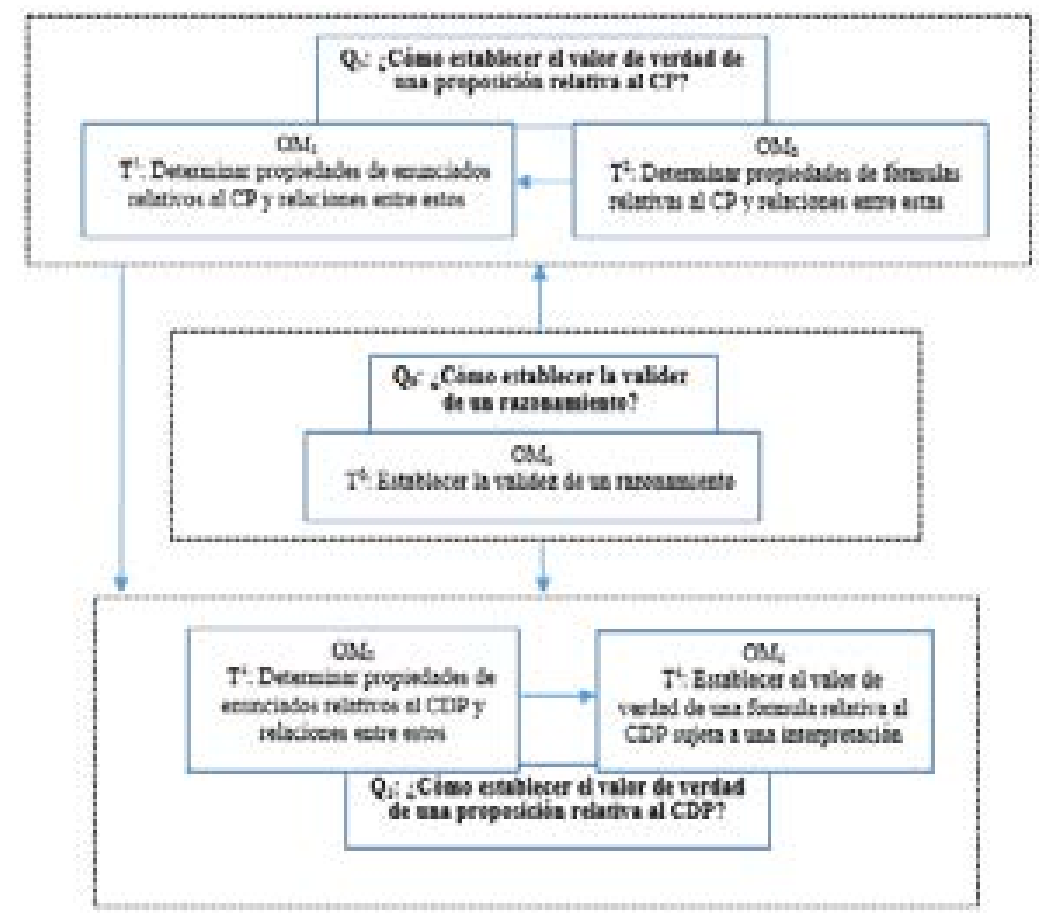

Imagen 1. Esquema general MPR

Fuente: Elaboración propia 
A $Q_{0}$, cuestión generatriz principal del MPR, se asocia $\mathrm{OM}_{0}$, y a esta última se vincula el tipo de tareas $T^{0}$ : Establecer la validez de un razonamiento. Los interrogantes $Q_{1}$ y $Q_{2}$ surgen de $Q_{0}$, y dan lugar al estudio del CP y el CDP de manera respectiva.

La cuestión $Q_{1}$ : ¿Cómo establecer el valor de verdad de una proposición relativa al CP? da origen al estudio del tipo de tareas asociadas a la $\mathrm{OM}_{1}$ y a la $\mathrm{OM}_{2}$. $\mathrm{OM}_{1}$ se caracteriza por el tipo de tareas $T^{1}$ : Determinarpropiedades de enunciados relativos al CP y relaciones entre estos; se comprende por enunciado, a una expresión del lenguaje cotidiano de la cual puede afirmarse que es verdadera o falsa pero no las dos a la vez. $\mathrm{OM}_{2}$ se identifica con el tipo de tareas $T^{2}$ : Determinar propiedades de fórmulas relativas al CP y relaciones entre estas. Las fórmulas son aquellas expresiones compuestas por letras que representan variables proposicionales, símbolos que representan conectivos y, si es el caso, términos de agrupación útiles para evitar ambigüedades.

El interrogante $Q_{2}$ : ¿Cómo establecer el valor de verdad de una proposición relativa al CDP? da lugar al tipo de tareas vinculadas a la $\mathrm{OM}_{3}$ y a la $\mathrm{OM}_{4}$. $\mathrm{OM}_{3}$ se relaciona con el tipo de tareas $T^{3}$ : Determinar propiedades de enunciados relativos al CDP y relaciones entre estos; y $\mathrm{OM}_{4}$ está representada por el tipo de tareas $T^{4}$ : Establecer el valor de verdad de una fórmula relativa al CDP sujeta a una interpretación.

$\mathrm{OM}_{0}$ es la praxeología que se desprende de la cuestión generatriz principal del MPR, de esta emergen las praxeologías $\mathrm{OM}_{1}, \mathrm{OM}_{2}, \mathrm{OM}_{3}$ y $\mathrm{OM}_{4}$. Asimismo, de cada una de estas cuatro OM emerge una red de praxeologías particular que no son descritas en el presente trabajo teniendo en cuenta su extensión.

Los tipos de tareas asociados a las OM antes citadas se vinculan a 15 géneros de tareas.
En la construcción de la $\mathrm{OM}_{1}$ se proponen tipos de tareas que se corresponden con los géneros Determinar, Establecer, Conectar, Construir, Representar y Negar; al abordar la $\mathrm{OM}_{2}$ se identifican tipos de tareas correspondientes a los géneros Determinar, Representar, Conectar, Construir, Establecer, Construir, Inferir, Examinar y Caracterizar, en la formulación de la $\mathrm{OM}_{3}$ se consideran tipos de tareas que se identifican con los géneros Determinar, Asociar, Conectar, Construir, Transformar, Representar, Establecer y Negar, y al concebir la $\mathrm{OM}_{4}$ se identificaron tipos de tareas asociados a los géneros de tareas: Establecer, Asociar, Determinar, Designar, Conectar, Construir, Convertir, Establecer, Transformar y Negar. En particular, el género de tareas Determinar refiere a aquellas tareas en las que se hacen precisiones con base en información conocida; el género Establecer se emplea para aludir a tareas en las cuales se requiere verificar o confirmar determinadas características de una proposición; los quehaceres correspondientes al género Conectar hacen referencia a tareas que implican unir proposiciones de forma que se formen otras más complejas; el género Construir reúne tareas que demandan del uso organizado de herramientas y reglas para concebir un propósito; al género Representar se asocia con tareas que demandan del uso de símbolos para presentar enunciados; el género Negar hace alusión a tareas que implican aseverar que una proposición no es cierta; al género Inferir se vincula a aquellas tareas orientadas a obtener una conclusión a partir de un conjunto de premisas; aquellas tareas relacionadas con escudriñar con diligencia y atención un concepto se vinculan al género Examinar, al género Caracterizar se asocia a tipos de tareas en las que se determinan los atributos que distinguen un sistema; el género Asociar hace alusión a tareas tendientes a juntar dos componentes de un sistema con un objetivo determinado; al género Designar alude a tareas que suponen destinar algo 
para un fin determinado; el género de tareas Demostrar engloba las tareas que requieren probar de manera deductiva un argumento con el fin de asegurar la verdad de una proposición matemática; al género Precisar se asocian tareas relacionadas con realizar una descripción de manera rigurosa; el género Intercambiar hace alusión a tareas que posibilitan, con base en una proposición compuesta por uno de los dos cuantificadores, construir una equivalente, que incluye el otro cuantificador; y aquellas tareas en las que se les da significado a variables proposicionales que componen fórmulas se vinculan al género Convertir. Es importante resaltar que el MPR constituyó una herramienta fundamental para analizar y cuestionar las características de la OMPE. Este análisis se realiza en el siguiente apartado.

\section{LA ORGANIZACIÓN MATEMÁTICA PROPUESTA A ENSEÑAR}

La reconstrucción de la OMPR demandó la confección de la tabla indicada en la Tabla 1, a partir del material propuesto para el estudio por ambos profesores. Este estudio demandó el análisis de 1716 tareas. Se destaca que las tareas propuestas en el libro de texto se vinculan a los géneros de tareas que se indican a continuación, y definidos de la misma manera que se propuso en el MPR: Representar, Establecer, Identificar, Demostrar, Inferir, Construir, Determinar, Convertir, Completar, Negar, Relacionar y Corregir. Desagregando por cantidad de tareas formuladas por cada género, se pudo establecer que: 447 se vinculan a Representar, 343 se asocian a Establecer, 337 corresponden al género Identificar, a Demostrar se vinculan 229 tareas, 180 aluden a Inferir, 59 se vinculan a Construir, 37 quehaceres refieren a Determinar, 35 se asocian al género Convertir, 21 tareas corresponden a Completar, 12 hacen referencia a Negar, 8 aluden a Relacionar y al género Corregir se vinculan 8 tareas. Es decir, el género más típico es Representar con 447 tareas asociadas; y aquellos géneros a los que se vincula un menor número de tareas son Relacionar y Corregir con 8 tareas asociadas a cada uno.

Se destaca que se observó diferencias entre los géneros de tareas identificados en la OMPE y los formulados en el MPR. Las tareas correspondientes a los géneros Completar y Relacionar identificados en la OMPE no se incluyen en el MPR debido a que se consideran quehaceres de tipo memorístico, que no aportan al hacer matemático. En cada tarea asociada al género Completar, se presentan las definiciones de algunas nociones, pero incompletas, y se demanda indicar la palabra que al ser ubicada en cada espacio en blanco concluye la definición; para el caso de las tareas asociadas al género Relacionar, se presentan dos columnas, la del lado izquierdo se compone de nociones, la del lado derecho está conformada por proposiciones, para resolver la tarea, se solicita vincular cada palabra de la columna izquierda con una proposición de la columna de la derecha. Por su parte, las tareas asociadas al género Identificar no se incorporan en el MPR, porque se considera innecesario, debido a que este instrumento incluye el género Construir, $\mathrm{y}$ al construir proposiciones, necesariamente se están identificando las componentes de estas; además, el género Construir posibilita la formulación de tareas funcionales para la solución de problemas. De igual forma, Corregir no se toma en cuenta como género en el MPR porque trata sobre la detección y corrección de errores en procesos deductivos, y el género Demostrar, incluido en el MPR, trata sobre probar de manera deductiva argumentos; y se estima que si se puede demostrar, en consecuencia, se pueden detectar y corregir errores en demostraciones. Por otra parte, 6 géneros de tareas considerados en el MPR no forman parte de la OMPE. Los géneros Conectar y Asociar son útiles para la construcción de proposiciones moleculares y fórmulas relativas 
al CP y al CDP; variados problemas tanto de carácter intramatemático como extramatemático demandan de la construcción de proposiciones y de fórmulas. Los géneros Examinar y Caracterizar tienen que ver con el estudio acerca de notaciones alternativas para abordar el CP y el CDP, correspondientes a simbologías que posibilitan un estudio más profundo de estos ámbitos de la lógica matemática. Designar y Transformar son géneros que involucran tipo de tareas fundamentales que se emplean al estudiar el CDP; el primero es útil al hacer referencia a los términos que componen las proposiciones, y el segundo pone de presente la estrecha relación entre el cuantificador universal y el cuantificador existencial, los que permiten resolver tanto problemas matemáticos como problemas de la vida cotidiana.

Las técnicas explícitas en el texto, indicadas para algunos ejemplares de tareas, permitió hacer inferencias acerca de la vinculación de las tareas con los indicadores matemáticos de completitud. En la Imagen 2 se indica un ejemplar de tarea resuelto en el libro de texto analizado.

Ahora que se conocea ya dos reglas de inferencia se pueden hacer demostraciones cortas que requieran el uso de arobas. Considérese el ejemplo que sigue en el que el modus ponendo ponens, PP, y la doble negación, DN, se utilizan para llegar a la conclusión:
(1) $P \rightarrow Q$
P
(2) $P$
$\mathrm{P}$
(3) $Q$
PP 1, 2
(4) $\neg \neg Q$
DN 3

En la demostración hay dos premisas y dos líneas derivadas. La linea (3) se deriva de las líneas (1) y (2) por el modus ponendo ponens. La línea (4) se deduce de la línea (3) por la regla de la doble negación.

Imagen 2. Ejemplar resuelto en el libro de texto

Fuente: Suppes y Hill (1975)

El ejemplar exhibido en la Imagen 2 se asocia al género de tareas Demostrar y al tipo de tareas Demostrar la conclusión producto de un conjunto de premisas representadas simbólicamente. Se empleó una única técnica para solucionar la tarea, esta implica la aplicación de las reglas de inferencia PP (modus ponendo ponens) y DN (doble negación) a partir de las premisas, hasta llegar a la conclusión. El entorno tecnológico-teórico empleado lo componen los conceptos regla modus ponendo ponens y regla doble negación. El quehacer se vincula únicamente al indicador de completitud OML7 propuesto por Fonseca (2004), dado que se materializa una integración de nociones que componen el entorno tecnológico-teórico.

El análisis de los ejemplares de tareas resueltos en el material, producto de la información recogida en la última columna de la Tabla 1, evidenció que la gran mayoría de tareas se vincula al indicador OML7 porque presentan integración de elementos del entorno tecnológico teórico. Solamente algunos quehaceres se vinculan al indicador OML1 puesto que presentan integración de tipos de tareas. En contadas excepciones se presentan tareas asociadas a los indicadores 
OML2 y OML4, dado que algunas veces se emplea más de una técnica para resolver los quehaceres, y en ocasiones se estudian tareas inversas; por ejemplo, se identificaron algunas tareas vinculadas al tipo de tarea Representar proposiciones simbólicamente, y otras asociadas al tipo de tarea Representar proposiciones en lenguaje cotidiano; tareas referidas a los tipos anteriores son inversas, puesto que para resolverlas demandan del empleo de técnicas inversas; en el primer caso se propone una proposición en lenguaje cotidiano y se solicita representarla mediante símbolos, en el segundo caso se formula una proposición en lenguaje simbólico y se demanda representarla en lenguaje cotidiano. No se identificaron tareas asociadas a los indicadores OML3, OML5, OML6 y OML8. Esto último indica que las técnicas tienden a identificarse con los objetos ostensivos utilizados para representarlas; no se analizó el funcionamiento y el resultado de la aplicación de las técnicas; no se tuvieron en cuenta tareas abiertas, las que son particularmente útiles para resolver problemáticas que aquejan a las personas en la vida cotidiana; y tampoco se modificaron las hipótesis del sistema ni se perturbaron situaciones iniciales del problemas con el fin de enriquecer el proceso de estudio.

Para el caso de las tareas propuestas para ser resueltas por los estudiantes, se encontró que en estas se exige aplicar las mismas técnicas que se proponen en los ejemplares resueltos, y solo permiten reutilizar técnicas pero no cuestionarlas y modificarlas. De igual forma, en la gran mayoría de los casos el enunciado de la tarea indica la técnica a emplear para resolverla. Además, la gran mayoría de tareas se asocia al indicador OML7; algunas de ellas se asocian al indicador OML1, y en contadas excepciones se encuentran tareas relacionadas con los indicadores OML2 y OML4; no se encontraron tareas asociadas a los indicadores OML3, OML5, OML6 y OML8. Se observa que las tareas resultas y las tareas propuestas para su resolución en el libro de texto presentan una vinculación idéntica con los indicadores de completitud propuestos por Fonseca (2004) y Lucas (2010). En la Tabla 2 que se presenta a continuación, se expone una tarea propuesta en el libro de texto para ser resuelta por los estudiantes, con su respectiva solución, propuesta por los investigadores, en función de las técnicas y el bloque tecnológico-teórico propuesto en el texto. La tarea se describe de acuerdo a las categorías descritas en la Tabla 1. 
Tabla 2. Tarea propuesta en el libro de texto para su resolución y las técnicas para su hacer

\begin{tabular}{|c|c|c|c|c|c|}
\hline $\begin{array}{c}\text { Género } \\
\text { de } \\
\text { tareas }\end{array}$ & $\begin{array}{l}\text { Tipo de } \\
\text { tareas }\end{array}$ & Ejemplar y solución & Técnica & $\begin{array}{c}\text { Bloque } \\
\text { tecnológico- } \\
\text { teórico }\end{array}$ & IMC \\
\hline Inferir & $\begin{array}{l}\text { Inferir una } \\
\text { conclusión } \\
\text { a partir de } \\
\text { un conjunto } \\
\text { de premisas } \\
\text { representadas } \\
\text { simbólicamente }\end{array}$ & $\begin{array}{l}\text { Usar la Ley de silogismo } \\
\text { disyuntivo (SD) para } \\
\text { obtener una conclusión } \\
\text { del siguiente conjunto de } \\
\text { premisas. } \\
\text { (1) }(\mathrm{R \& S}) \mathrm{vT} \quad \mathrm{P} \\
\text { (2) }(\mathrm{R \& S}) \rightarrow \sim \mathrm{P} \\
\text { (3) } \mathrm{T} \rightarrow \mathrm{P} \\
\text { Solución: } \mathrm{P} \\
\text { (4) pv Q SD } \\
1,2,3\end{array}$ & $\begin{array}{l}\text { Verificar } \\
\text { relaciones entre } \\
\text { las premisas y } \\
\text { aplicar la regla } \\
\text { de inferencia } \\
\text { Ley de silogismo } \\
\text { disyuntivo; } \\
\text { con lo cual se } \\
\text { obtiene una } \\
\text { conclusión. }\end{array}$ & $\begin{array}{l}\text { Proposición y } \\
\text { Ley de silogismo } \\
\text { disyuntivo }\end{array}$ & OML7 \\
\hline
\end{tabular}

Fuente: Elaboración propia

En las dos primera columnas que conforman la Tabla 2 se puede apreciar que el ejemplar se asocia al género de tareas Inferir y al tipo de tareas Inferir una conclusión a partir de un conjunto de premisas representadas simbólicamente. En la tercera columna se presenta la resolución de la tarea realizada por los investigadores. En el enunciado de la tarea se pone de presente que se indica la manera de resolverla, se solicita usar la Ley de silogismo disyuntivo. En las columnas cuarta y quinta se describen respectivamente, la técnica utilizada para resolver la tarea y las nociones del entorno tecnológico-teórico empleadas. $\mathrm{Y}$ en la sexta columna se resalta que la tarea considerada se asocia únicamente al indicador de completitud
OML7 propuesto por Fonseca (2004).

En el Anexo de este trabajo, se exhibe una tabla en la que se indican las técnicas y el entorno tecnológico-teórico que justifica el hacer propuesto de 12 tareas identificadas en el libro de texto, formuladas para la resolución por parte de los estudiantes. Cada tarea corresponde a uno de los 12 géneros identificados en la reconstrucción de la OMPE, y se analizaron empleando las categorías descritas en la Tabla 1. 


\section{CONCLUSIONES}

En este trabajo se presentan resultados del estudio de la completitud de la OMPE en torno al CP y al CDP para la formación de estudiantes para profesor en matemática. La reconstrucción de la OMPE se la realizó a partir de un libro de texto recomendado para el estudio por ambos profesores. El estudio también demandó el diseño de un MPR, constituyendo una herramienta central para analizar el material propuesto por los profesores para el estudio. Cabe resaltar que se observó diferencias entre los géneros de tareas identificados en la OMPE y los propuestos en el MPR. Las tareas asociadas a los géneros Completar y Relacionar identificados en la OMPE no son tenidas en cuenta en el MPR puesto que se consideran quehaceres de tipo memorístico; los géneros Identificar y Corregir no forman parte del MPR, debido a que otros géneros contemplados en este último modelo incluyen los tipos de tareas asociadas a los dos géneros antes mencionados. En cambio, los géneros Conectar, Asociar, Examinar, Caracterizar, Designar y Transformar que componen el MPR no forman parte de la OMPE, a pesar de su importancia para la construcción de proposiciones moleculares y de fórmulas relativas al CP y al CDP. Se pudo establecer que, las tareas resueltas en el material analizado y las tareas formuladas para ser resueltas por los estudiantes, presentan una vinculación similar con los indicadores de completitud, y se caracterizan por conformar una OM con bajo grado de completitud, debido a que se asocian solamente a cuatro de los ocho indicadores propuestos por Fonseca (2004) y Lucas (2010). Se identificaron tareas que solo se vinculan con los indicadores OML1, OML2, OML4 y OML7; además, la vinculación con los indicadores OML2 y OML4 es muy débil, debido a que un escaso número de tareas se asocian a estos.
Es posible afirmar que en las tareas resultas y en las tareas propuestas para su resolución en el material analizado, se evidenció integración de tipos de tareas e integración de elementos del entorno tecnológico-teórico; también, en algunos casos se advirtió el empleó de más de una técnica en la solución de tareas, y se abordaron tareas inversas. En contraste, estas tareas se caracterizan por estar asociadas a técnicas rígidas y desarticuladas entre sí; no se observaron instancias que permitan elegir la representación ostensiva más adecuada para representar técnicas particulares; no se evidenció interpretación del funcionamiento y el resultado de la aplicación de las técnicas; no se estudiaron tareas abiertas, las que por sus características, son de utilidad en la solución de problemas de la vida cotidiana; tampoco se consideró perturbar la situación inicial o modificar la hipótesis del sistema para estudiar casos diferentes y así ampliar y completar el proceso de estudio. Por otro lado, además de la reconstrucción del MPR y de la OMPE, resulta de interés analizar la reconstrucción de la OMEE (Organización Matemática Efectivamente Enseñada); lo que permite realizar las comparaciones respectivas entre los tres constructos mencionados (MPR, OMPE y OMEE). Dichos constructos hacen posible tomar conocimiento acerca de las prácticas llevadas a cabo por docentes que forman a estudiantes de profesorado en matemática, y constituyen una base fundamental para proponer tareas que contribuyan a la reconstrucción de OM con alto grado de completitud, útiles para la enseñanza funcional de temas relativos a CP y CDP. 


\section{REFERENCIAS BIBLIOGRÁFICAS}

Barquero, B., Bosch, M. y Gascón, J. (2013). Las tres dimensiones del problema didáctico de la modelización matemática. Educ. Matem. Pesq., Sao Paulo. 15(1), 1-28.

Bosch, M. y Gascón, J. (2007). 25 años de Transposición didáctica. En L. RuizHigueras, A. Estepa, F. J. García (Eds.) Sociedad, Escuela y Matemáticas. Aportaciones de la Teoría antropológica de lo didáctico (pp. 385-406). Jaén: Publicaciones de la Universidad de Jaén.

Cardona Hurtado, O. A. y Corica, A. R. (2020). Estudio sobre las praxeologías relacionadas con cálculo proposicional y cálculo de predicados en la formación de futuros profesores de matemática. UNIÓN - Revista Iberoamericana de Educación Matemática, 16(60), 260-280.

Copi, I. y Cohen, C. (2013). Introducción a la lógica. México: Limusa.

Chevallard, Y. (1991). La transposición didáctica. Del saber sabio al saber enseñado. Buenos Aires, Argentina: Aique.

Chevallard, Y. (1997). La transposición didáctica. Del saber sabio al saber enseñado. Aique, Buenos Aires.

Chevallard, Y. (1999). L'analyse des pratiques enseignantesen théorie anthropologique du didactique. Recherches en didactique des mathématiques, 19(2), 221-266.

Del Callejo, D.; Del Callejo, E. y Canal, M. (2016). Lógica: el pensamiento matemático. México: Textos universitarios, Universidad Veracruzana.
Fernández, G. y Sáez, F. (1995). Fundamentos de informática. Madrid, España: Ediciones Anaya multimedia S.A.

Fonseca, C. (2004). Discontinuidades matemáticas y didácticas entre la enseñanza secundaria y la enseñanza universitaria. Tesis de doctorado no publicada, Universidad de Vigo. España.

García, F. y Sierra, T. (2015) Modelos epistemológicos de referencia en el análisis de la actividad matemática en libros de texto: El caso del número en la escuela infantil. En c. Fernández, M. Molina y N. Planas (eds), Investigación en educación matemática XIX (pp. 299307). Alicante: SEIEM.

García, F., Barquero, B., Florensa, I. y Bosch, M. (2019). Diseño de tareas en el marco de la Teoría Antropológica de lo Didáctico. Avances de Investigación en Educación Matemática, 15, 75-94.

Garrido, M. (2005). Lógica simbólica. Madrid, España: Editorial Tecnos.

Gascón, J. (1998). Evolución de la didáctica de las matemáticas como disciplina científica. Recherches en Didactique des Mathématiques, 18/1(52), 7-33.

Gascón, J. (2014). Los modelos epistemológicos de referencia como instrumentos de emancipación de la didáctica y la historia de las matemáticas. Educación matemática. 26(1), 99-123.

Hernández, R.; Fernández, C. y Baptista. P. (2014). Metodología de la Investigación. $6^{\circ}$ edición. Mc Graw-Hill Interamericana Editores: Ciudad de México.

Lucas, C. (2010). Organizaciones matemáticas locales relativamente completas. Tesis de doctorado no publicada, Universidad de Vigo. España. 
Otero, M. R.; Fanaro, M. A.; Corica, A. R.; Llanos, V. C.; Sureda, P. y Parra, V. (Ed). (2013). La Teoría Antropológico de lo Didáctico en el Aula de Matemática. Buenos Aires: Editorial DUNKEN.

Suppes, P. y Hill, S. (1975). Introducción a la lógica matemática. Editorial Reverté S.A.: Cuauhtémoc, México.

Vásquez, M. (2020). Utilización del Kahoot para la introducción de la lógica proposicional en la E.S.O. Épsilon - Revista de Educación Matemática, 106, 61-68.

\section{ANEXO}

Tareas identificadas en la reconstrucción de la OMPE

\begin{tabular}{|c|c|c|c|c|c|}
\hline $\begin{array}{c}\text { Género de } \\
\text { tareas }\end{array}$ & $\begin{array}{l}\text { Tipo de } \\
\text { tareas }\end{array}$ & Ejemplar y solución & Técnica & \begin{tabular}{|c|} 
Bloque \\
tecnológico- \\
teórico
\end{tabular} & I MC \\
\hline Negar & $\begin{array}{l}T_{6}^{P}: \\
\text { Negar : } \\
\text { proposiciones } \\
\text { relativas al CP }\end{array}$ & $\begin{array}{l}\text { Dar la negación de cada una } \\
\text { de las proposiciones siguientes } \\
\text { añadiendo símbolos de } \\
\text { negación, y paréntesis si es } \\
\text { necesario. } \\
\text { Ítem: } \\
\mathrm{T}_{\mathrm{V}} \neg \mathrm{S} \\
\text { Solución: } \\
\neg\left(\mathrm{T}_{\mathrm{v}} \neg \mathrm{S}\right)\end{array}$ & $\begin{array}{l}\text { Encerrar entre } \\
\text { paréntesis la } \\
\text { proposición } \\
\text { molecular, y luego } \\
\text { ubicar antes del } \\
\text { paréntesis el } \\
\text { símbolo de la } \\
\text { negación. }\end{array}$ & $\begin{array}{l}\text { Proposición, } \\
\text { proposición } \\
\text { atómica, } \\
\text { proposición } \\
\text { molecular, } \\
\text { término } \\
\text { de enlace, } \\
\text { símbolos } \\
\text { auxiliares y } \\
\text { negación }\end{array}$ & $\begin{array}{l}\text { OML1 } \\
\text { Y } \\
\text { OML7 }\end{array}$ \\
\hline
\end{tabular}




\begin{tabular}{|c|c|c|c|c|c|}
\hline Relacionar & \begin{tabular}{|l}
$T_{7}:$ \\
Relacionar \\
cada enunciado \\
de la columna \\
izquierda \\
con uno de \\
la columna \\
derecha
\end{tabular} & $\begin{array}{l}\text { Aparear cada una de las } \\
\text { palabras de la izquierda con los } \\
\text { ejemplos o definiciones en la } \\
\text { lista de la derecha. } \\
\text { Ítems y solución: } \\
\\
\end{array}$ & \begin{tabular}{|l|} 
Examinar las \\
expresiones \\
de la columna \\
izquierda; luego, \\
para cada una de \\
ellas buscar una \\
expresión de la \\
columna derecha \\
que tenga algún \\
tipo de relación, y \\
unirlas.
\end{tabular} & $\begin{array}{l}\text { Proposición, } \\
\text { proposición } \\
\text { atómica, } \\
\text { proposición } \\
\text { molecular, } \\
\text { término } \\
\text { de enlace, } \\
\text { símbolos } \\
\text { auxiliares, } \\
\text { término } \\
\text { de enlace } \\
\text { dominante, } \\
\text { conjunción, } \\
\text { disyunción, } \\
\text { implicación y } \\
\text { negación }\end{array}$ & OML7 \\
\hline Completar & $\begin{array}{l}T_{P}: \\
\\
\text { Completar la } \\
\text { proposición } \\
\text { indicando } \\
\text { la noción } \\
\text { requerida para } \\
\text { dar sentido a la } \\
\text { afirmación }\end{array}$ & $\begin{array}{l}\text { Completar las proposiciones } \\
\text { siguientes eligiendo de entre las } \\
\text { palabras escritas al final la que } \\
\text { está definida por la proposición } \\
\text { dada. } \\
\text { Ítem: } \\
\text { La proposición molecular que } \\
\text { utiliza el termino de enlace " } y \text { " es } \\
\text { una }\end{array}$ & $\begin{array}{l}\text { Elegir de la lista } \\
\text { dada la palabra } \\
\text { adecuada que } \\
\text { de sentido a } \\
\text { la expresión, y } \\
\text { escribirla en el } \\
\text { espacio en blanco. }\end{array}$ & $\begin{array}{l}\text { Proposición, } \\
\text { proposición } \\
\text { atómica, } \\
\text { proposición } \\
\text { molecular, } \\
\text { término } \\
\text { de enlace, } \\
\text { símbolos } \\
\text { auxiliares, } \\
\text { término } \\
\text { de enlace } \\
\text { dominante, } \\
\text { conjunción, } \\
\text { disyunción, } \\
\text { implicación, } \\
\text { negación, }\end{array}$ & OML7 \\
\hline
\end{tabular}




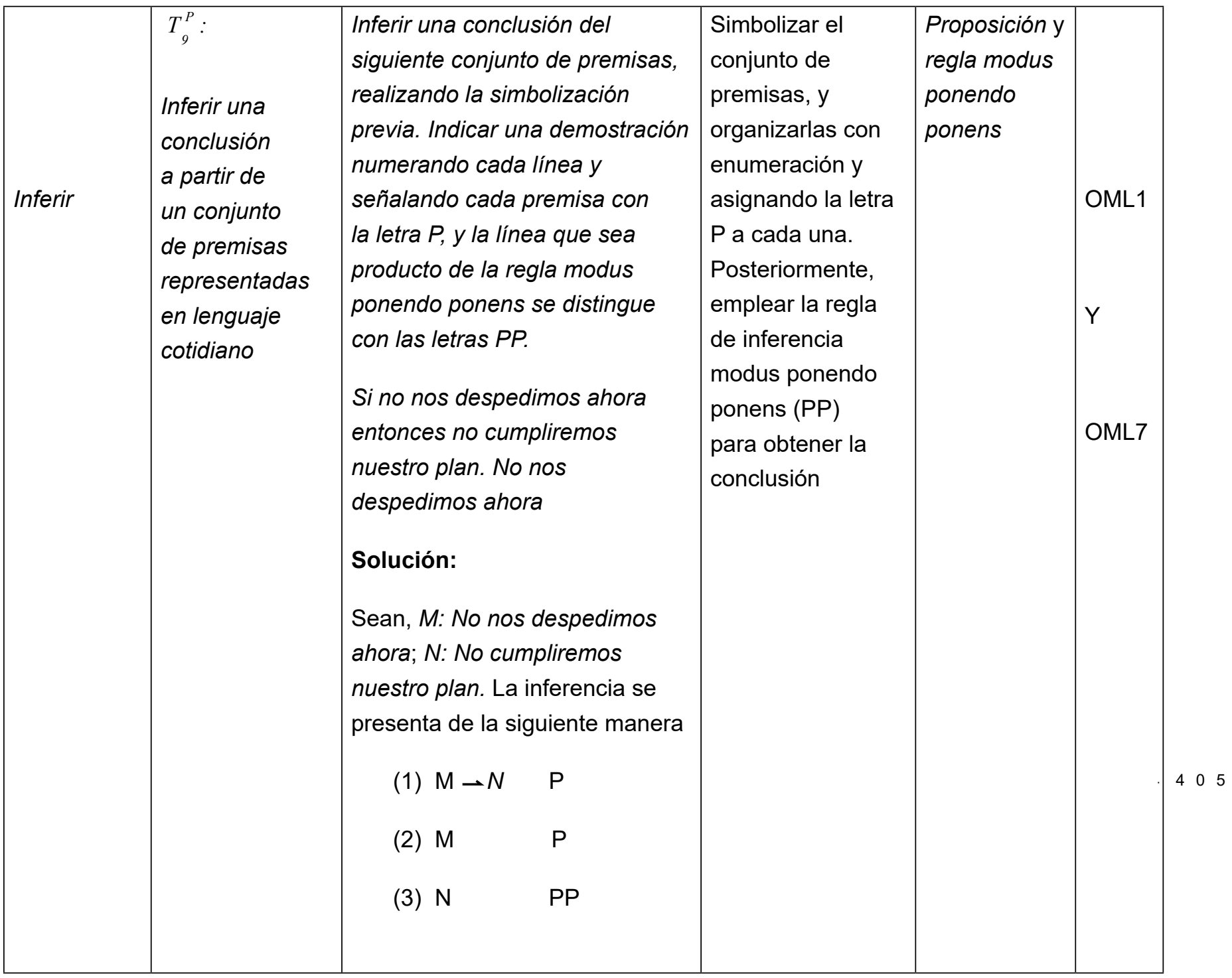




\begin{tabular}{|c|c|c|c|c|c|}
\hline Identificar & $\begin{array}{l}T_{5}^{P}: \\
\text { Identificar } \\
\text { el término } \\
\text { de enlace } \\
\text { dominante en } \\
\text { una proposición }\end{array}$ & 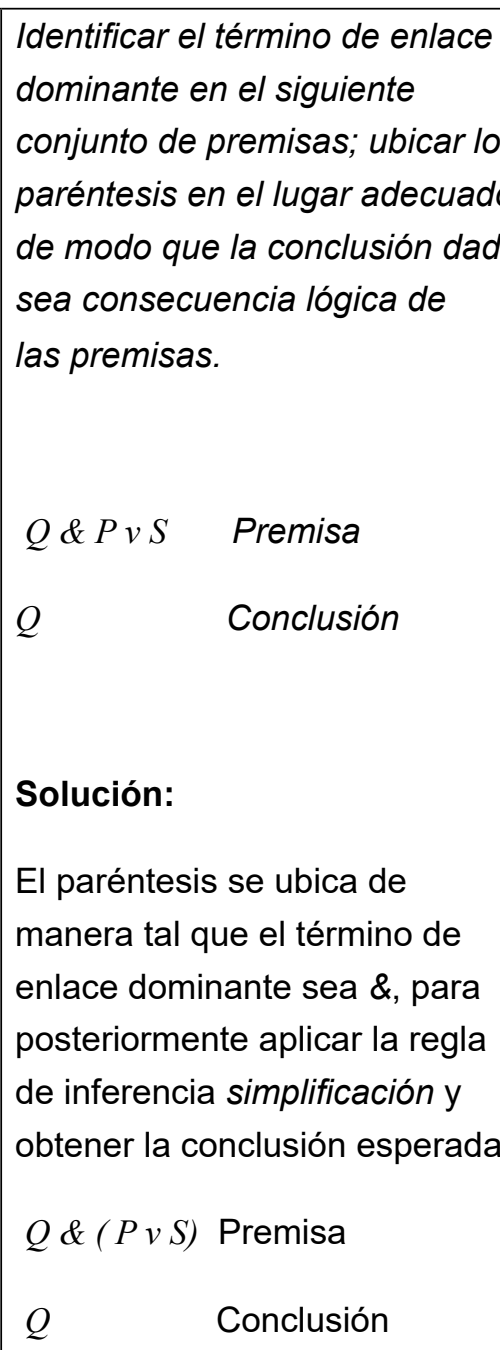 & $\begin{array}{l}\text { Identificar la } \\
\text { premisa y la } \\
\text { conclusión. En } \\
\text { vista de que } \\
\text { en la premisa } \\
\text { está presente } \\
\text { la el termino de } \\
\text { enlace \& y la } \\
\text { conclusión Q, } \\
\text { entonces resulta } \\
\text { adecuado ubicar } \\
\text { los paréntesis } \\
\text { de tal forma que } \\
\& \text { sea el termino } \\
\text { dominante, para } \\
\text { que mediante la } \\
\text { regla de inferencia } \\
\text { simplificación, la } \\
\text { concusión sea } \\
\text { consecuencia } \\
\text { lógica de la } \\
\text { premisa. }\end{array}$ & $\begin{array}{l}\text { Proposición, } \\
\text { término } \\
\text { de enlace } \\
\text { dominante } \\
\text { y regla } \\
\text { simplificación }\end{array}$ & OML7 \\
\hline
\end{tabular}




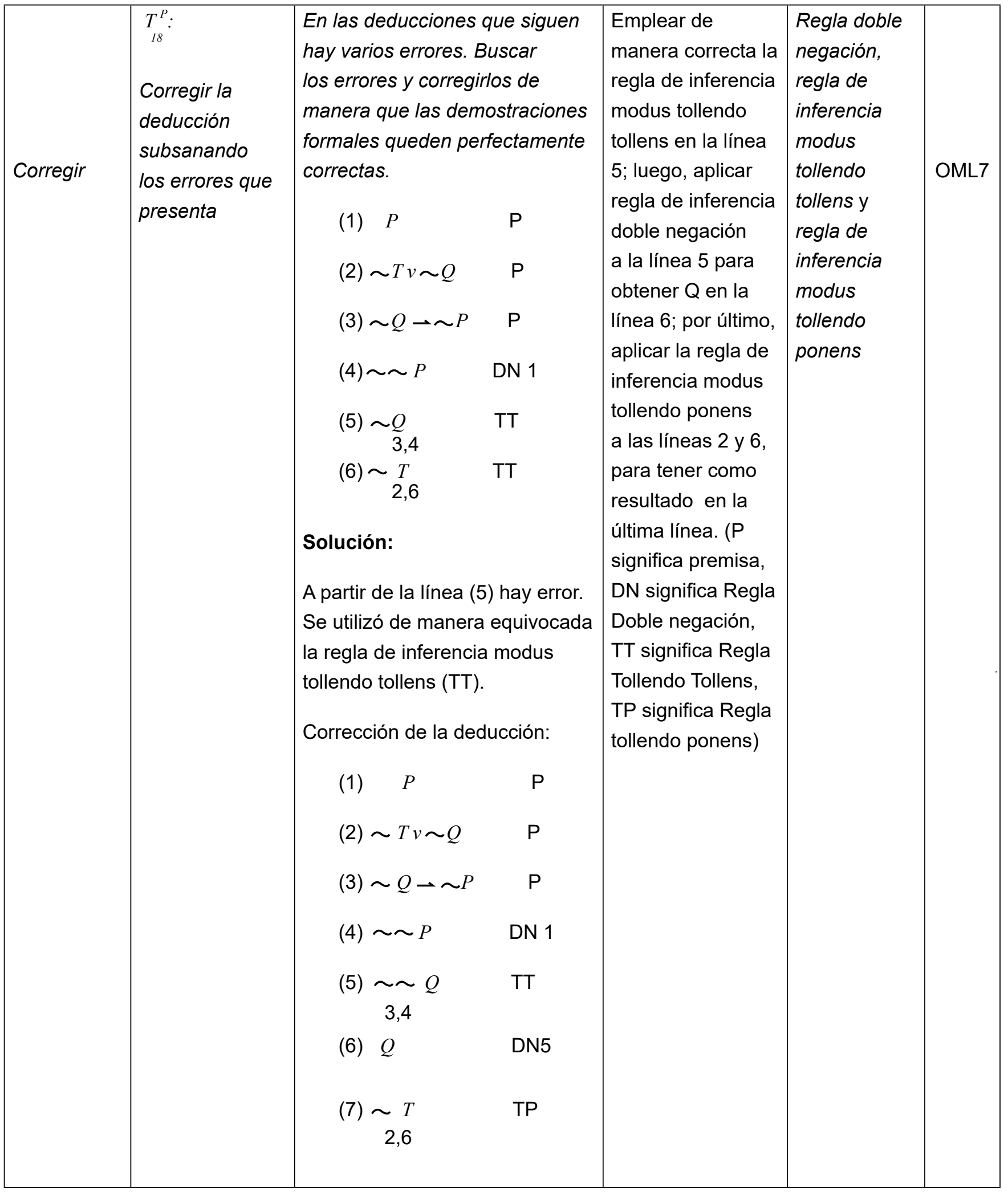




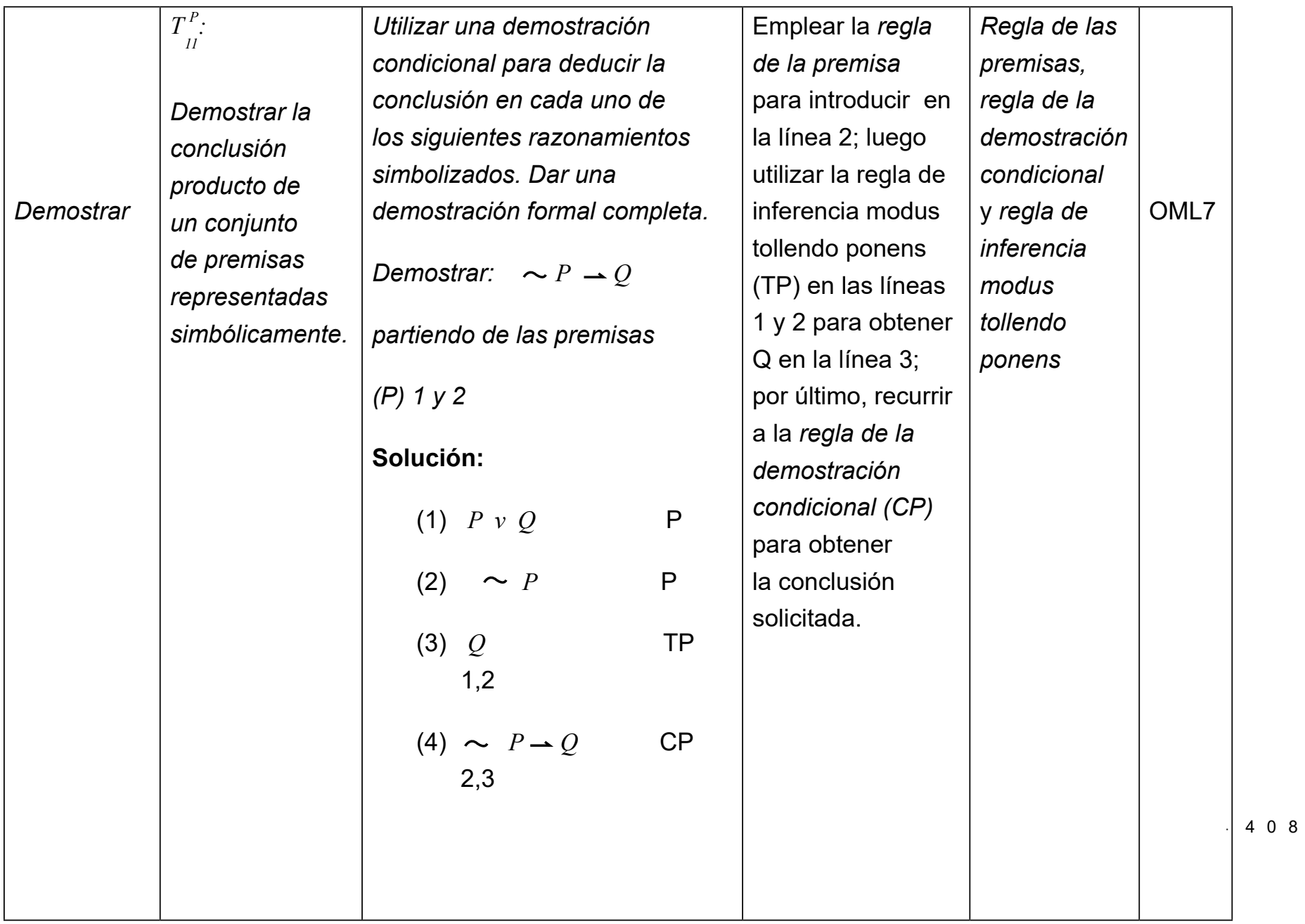




\begin{tabular}{|c|c|c|c|c|c|c|c|c|c|}
\hline Establecer & $\begin{array}{l}T_{23}^{P}: \\
\text { Establecer la } \\
\text { equivalencia de } \\
\text { dos fórmulas }\end{array}$ & \multicolumn{5}{|c|}{$\begin{array}{l}\text { Utilizar tablas de certeza para } \\
\text { determinar para cada uno de } \\
\text { los pares de proposiciones } \\
\text { siguientes si son lógicamente } \\
\text { equivalentes. } \\
P v \sim Q \\
Q \rightarrow P \\
\text { Solución: } \\
\text { En las cuarta y quinta } \\
\text { columna de la siguiente tabla } \\
\text { se presentan los valores de } \\
\text { verdad asociados a las dos } \\
\text { proposiciones: }\end{array}$} & $\begin{array}{l}\text { Emplear una tabla } \\
\text { de verdad para } \\
\text { establecer los } \\
\text { valores de verdad } \\
\text { asociados a cada } \\
\text { proposición. Si } \\
\text { los valores de } \\
\text { verdad relativos a } \\
\text { las proposiciones } \\
\text { son exactamente } \\
\text { iguales, entonces } \\
\text { las proposiciones } \\
\text { son equivalentes, } \\
\text { en caso contrario } \\
\text { estas no son } \\
\text { equivalentes. }\end{array}$ & $\begin{array}{l}\text { Proposición } \\
\text { molecular } \\
\text { término } \\
\text { de enlace } \\
\text { dominante, } \\
\text { taba de } \\
\text { verdad de } \\
\text { la negación, } \\
\text { tabla de } \\
\text { verdad de la } \\
\text { disyunción, } \\
\text { tabla de } \\
\text { verdad de la } \\
\text { implicación, } \\
\text { tabla de } \\
\text { verdad y } \\
\text { equivalencia } \\
\text { tautológica }\end{array}$ & $\begin{array}{l}\text { OML1 } \\
\text { Y } \\
\text { OML7 }\end{array}$ \\
\hline
\end{tabular}




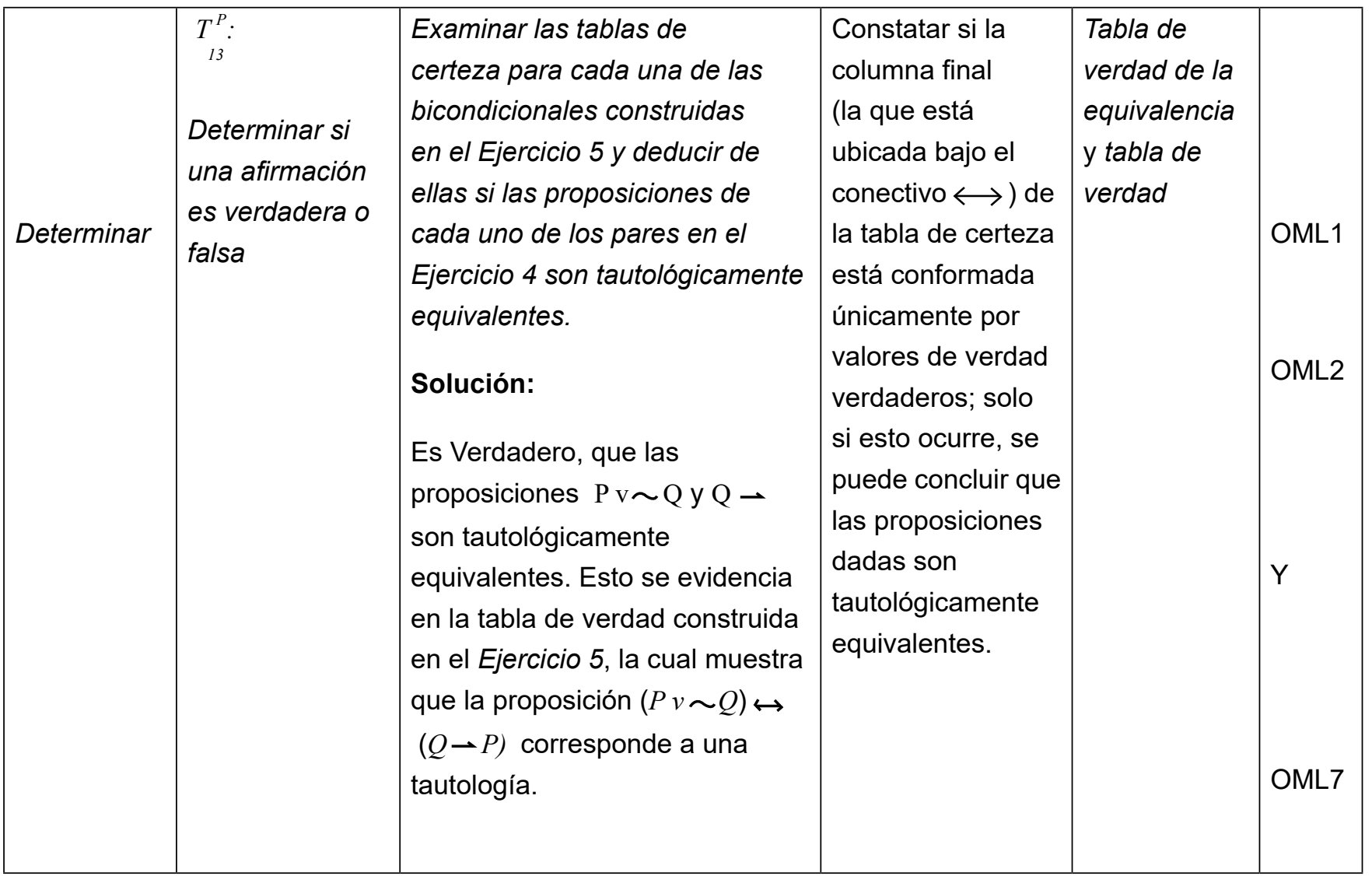




\begin{tabular}{|c|c|c|c|c|c|}
\hline Representar & $\begin{array}{l}T_{25}^{P}: \\
\\
\text { Representar } \\
\text { simbólicamente } \\
\text { proposiciones } \\
\text { relativas al CDP }\end{array}$ & $\begin{array}{l}\text { En cada una de las } \\
\text { proposiciones siguientes el } \\
\text { domino de referencia es el } \\
\text { conjunto de números. Usar el } \\
\text { cuantificador universal para } \\
\text { simbolizar las proposiciones } \\
\text { pero no utilizar letras } \\
\text { sustituyendo a los predicados. } \\
\text { Expresar las negaciones } y \\
\text { predicados con variables en } \\
\text { símbolos matemáticos típicos } \\
\text { o en el lenguaje usual (para el } \\
\text { Ejercicio 11, se indica simbolizar } \\
\text { los predicados). } \\
\text { Para cada } x, x \text { no es divisible } \\
\text { por cero. } \\
\text { Solución: } \\
\text { Sea, } \\
\text { Dx↔ X es divisible por cero } \\
\text { Luego, la simbolización } \\
\text { requerida es: } \\
(\forall x) \text { ( } D_{x} \text { ) } \\
\text { Que significa, ningún número es } \\
\text { divisible por cero. }\end{array}$ & $\begin{array}{l}\text { Utilizar el símbolo } \\
\text { del cuantificador } \\
\text { universal para } \\
\text { simbolizar para } \\
\text { cada } x \text {. Simbolizar } \\
\text { el predicado. Por } \\
\text { último, se presenta } \\
\text { la simbolización } \\
\text { de la proposición } \\
\text { propuesta en el } \\
\text { ítem. }\end{array}$ & $\begin{array}{l}\text { Negación, } \\
\text { término, } \\
\text { predicado, } \\
\text { variable, } \\
\text { fórmula } \\
\text { molecular, } \\
\text { cuantificador } \\
\text { universal y } \\
\text { dominio de } \\
\text { referencia }\end{array}$ & $\begin{array}{c}\text { OML1 } \\
\text { Y } \\
\text { OML7 }\end{array}$ \\
\hline
\end{tabular}




\begin{tabular}{|c|c|c|c|c|c|}
\hline Construir & \begin{tabular}{|l}
$T_{27}^{P}:$ \\
\\
Construir \\
fórmulas \\
relativas al CDP
\end{tabular} & $\begin{array}{l}\text { Construir fórmulas atómicas } \\
\text { utilizando variables. } \\
\text { Solución: } \\
\text { Considérese la fórmula } \\
\text { siguiente: } \\
(\forall x)(\sim T x) \\
\text { Es atómica porque no está } \\
\text { compuesta por términos de } \\
\text { enlace. Indica que ningún } \\
\text { elemento de un conjunto no } \\
\text { satisface la condición T. }\end{array}$ & $\begin{array}{l}\text { Establecer una } \\
\text { condición T, } \\
\text { que se asocia } \\
\text { al cuantificador } \\
\text { universal y a la } \\
\text { variable } x \text {. Luego, } \\
\text { escribir la fórmula } \\
\text { de manera que } \\
\text { tenga sentido. }\end{array}$ & $\begin{array}{l}\text { Cuantificador } \\
\text { universal, } \\
\text { variable, } \\
\text { negación, } \\
\text { predicado } \\
\text { y fórmula } \\
\text { atómica }\end{array}$ & OML7 \\
\hline Convertir & $\begin{array}{l}T_{28}^{P}: \\
\text { Convertir } \\
\text { fórmulas } \\
\text { relativas } \\
\text { al CDP en } \\
\text { proposiciones }\end{array}$ & $\begin{array}{l}\text { Convertir las fórmulas atómicas } \\
\text { del Ejercicio A en proposiciones } \\
\text { ciertas o falsas añadiendo } \\
\text { cuantificadores universales } \\
\text { utilizando el símbolo lógico. Para } \\
\text { Cada una de las proposiciones } \\
\text { resultantes decir si es cierta o } \\
\text { falsa. } \\
\text { x es un número mayor que } 4 \\
\text { Solución: } \\
\text { La fórmula asociada a la } \\
\text { proposición es } \\
x>4 \\
\text { Agregando el cuantificador } \\
\text { universal, se tiene } \\
\text { (Vx) (x }>4 \text { ) } \\
\text { La proposición anterior es falsa, } \\
\text { considerando como dominio } \\
\text { de referencia el conjunto de } \\
\text { números reales, dado que } \\
\text { existen números reales menores } \\
\text { que } 4 .\end{array}$ & $\begin{array}{l}\text { Escribir la fórmula } \\
\text { en lenguaje } \\
\text { simbólico. } \\
\text { Luego agregar } \\
\text { el cuantificador } \\
\text { universal para } \\
\text { convertirla en } \\
\text { proposición. Por } \\
\text { último, establecer } \\
\text { si la proposición } \\
\text { es verdadera o } \\
\text { falsa. }\end{array}$ & $\begin{array}{l}\text { Proposición } \\
\text { atómica } \\
\text { variable, } \\
\text { término, } \\
\text { predicado, } \\
\text { fórmula } \\
\text { atómica, } \\
\text { cuantificador } \\
\text { universal y } \\
\text { dominio de } \\
\text { referencia }\end{array}$ & OML7 \\
\hline
\end{tabular}

Fuente: Elaboración propia 